\title{
Effects of altered auditory feedback (AAF) on stuttering frequency during monologue speech production
}

\author{
Elena A. Antipova ${ }^{\mathrm{a}, \mathrm{b}, *}$, Suzanne C. Purdy ${ }^{\mathrm{a}}$, Marjorie Blakeley ${ }^{\mathrm{a}}$, Shelley Williams ${ }^{\mathrm{c}}$ \\ a Discipline of Speech Science, Department of Psychology, Tamaki Campus, 261 Morrin Road, Glen Innes, \\ The University of Auckland, Private Bag 92019, Auckland, New Zealand \\ b Oaklynn Special School, 20 Mayville Ave, New Lynn, Auckland, New Zealand \\ ${ }^{\mathrm{c}}$ Auckland District Health Board, Auckland, New Zealand
}

Received 22 September 2008; received in revised form 28 September 2008; accepted 28 September 2008

\begin{abstract}
The present study investigated the immediate effects of eight altered auditory feedback (AAF) parameters on stuttering frequency during monologue speech production on two occasions. One of the modern commercially available portable anti-stuttering devices, “The Pocket Speech Lab" (Casa Futura Technologies ${ }^{\circledR}$ ) was used in the study to produce the auditory feedback alterations. Six types of combined delayed auditory feedback (DAF) and frequency shifted auditory feedback (FAF) and two types of DAF alone were tested for eight participants aged 16-55 years, with stuttering severity ranging from mild to severe. The present study found that AAF is an effective means to reduce stuttering frequency during monologue speech production. All eight AAF experimental conditions reduced stuttering frequency, however, there was substantial variability in the stuttering reduction effect across experimental conditions and across participants. There was also instability in stuttering reduction across the two testing sessions. On average, a $75 \mathrm{~ms}$ time delay on its own and a combination of the $75 \mathrm{~ms}$ time delay and a half octave downward frequency shift were found to be more effective than other combinations of AAF parameters that were investigated.
\end{abstract}

Educational objectives: After reading this paper, the reader should be able to (1) summarize the research investigating the effect of altered auditory feedback on stuttering frequency during monologue speech production; (2) describe the stuttering reduction effect of the eight parameters of AAF tested during monologue speech production; and (3) discuss the possible clinical implications of the use of AAF for stuttering treatment.

(C) 2008 Elsevier Inc. All rights reserved.

Keywords: Stuttering; Altered auditory feedback; Delayed auditory feedback; Frequency shifted auditory feedback; Anti-stuttering device

\section{Introduction}

Auditory feedback is a term that describes the cortical processing mechanism for speech production that occurs when the produced message is compared and verified with the intended message while the speaker's auditory system perceives his or her own voice output (Howell, 2004; Ling, 2002). The term 'altered auditory feedback' (AAF) is used to refer to alterations to recurrent auditory information (Howell, 2004). There is substantial clinical and experimental data showing immediate fluency enhancing effects of AAF for people who stutter (Armson, Kiefte, Mason, \& De Croos,

\footnotetext{
* Corresponding author.

E-mail address: alexantip@yahoo.co.nz (E.A. Antipova).
} 
2006; Howell, 2004; Lincoln, Packman, \& Onslow, 2006). Although there is a large literature on the role of auditory feedback in speech production (Fairbanks, 1955; Howell \& Sackin, 2002; Lee, 1950; Max, Guenther, Gracco, Ghosh, \& Wallace, 2004; Postma, 2000; Postma \& Kolk, 1992; Venkatagiri, 2005), the mechanism of stuttering reduction with AAF in people who stutter is not completely understood. Alterations to auditory feedback are usually made using an external stimulus that is produced by another speaker, talking in unison, or using an electronic feedback device. There are a number of wearable commercially available devices that are intended to reduce stuttering frequency and severity, including the "Fluency Master", "Speech Easy", or "The Pocket Speech Lab".

There are three main forms of AAF used to decrease stuttering frequency: masked auditory feedback (MAF), delayed auditory feedback (DAF) and frequency shifted auditory feedback (FAF). MAF is the form of AAF whereby the speech of the speaker fed through headphones is constantly accompanied by an additional sound, usually noise. The masking noise is not necessarily mixed with a speech feedback signal. The speaker's air conducted voice signal is attenuated by virtue of the noise that is introduced. For DAF speakers hear their own speech with a short time delay. Delays are typically less than a quarter of a second (50-250 ms) (Burke, 1975; Kalinowski, Stuart, Sark, \& Armson, 1996; Van Borsel, Reunes, \& Van den Bergh, 2003). FAF is a form of AAF whereby the frequency range of the speaker's speech is shifted up or down leading to changes in pitch. FAF is quite a new form of auditory feedback alteration (Howell, El-Yaniv, \& Powell, 1987). Research on it is comparatively sparse, compared to the DAF and MAF literature. DAF and FAF have been shown to be more effective types of AAF than MAF (Howell et al., 1987; Kalinowski, Armson, Mieszkowski, Stuart, \& Gracco, 1993). There have been a few attempts to use a combination of DAF and FAF to improve their stuttering reduction effect (MacLeod, Kalinowski, Stuart, \& Armson, 1995; Stuart, Kalinowski, Rastatter, Saltuklaroglu, \& Dayalu, 2004). These attempts to intensify the effect of DAF and FAF by their combination did not result in additional reduction in stuttering.

Early experimental investigations on DAF focused on long delay intervals, in spite of findings by Lotzman (1961, cited in Howell et al., 1987) that a $50 \mathrm{~ms}$ delay is optimal for reduction in stuttering frequency. Long delays lead to reduced speech rate and prolongation of vowels, producing unnatural sounding speech (Goldiamond, 1965; Martin, Haroldson, \& Triden, 1984; Perkins, Bell, Johnson, \& Stochs, 1979). It has been suggested that reduced speech rate, rather than interference with an auditory feedback mechanism, causes the reduction in stuttering (Bloodstein, 1995; Costello-Ingham, 1993; Kalinowski et al., 1996; Wingate, 1970). Reduction in speech rate is known to improve fluency (Adams, Lewis, \& Besozzi, 1973; Perkins et al., 1979). Early AAF investigations highlighted a number of limitations of the technique, including the size of the devices, individual variability, lack of sustained improvements, and unnatural speech associated with long delays (Bloodstein, 1995; Costello-Ingham, 1993; Goldiamond, 1965; MacKay, 1968; Martin et al., 1984; Perkins et al., 1979). As a result, the effect of AAF on stuttering was discounted until a series of more recent experiments were published (Howell et al., 1987; Kalinowski et al., 1993; Kalinowski, Armson, \& Stuart, 1995; Kalinowski et al., 1996; Stuart, Kalinowski, Rastatter, \& Lynch, 2002). In contrast to the earlier studies, short delay intervals of 25, 50,75 ms were used. Stuttering frequency was investigated at fast and normal speech rates during unaltered and delayed auditory feedback (Kalinowski et al., 1993, 1995, 1996; Stuart et al., 2002). The main result of these studies was that slowing down of speech is not a critical factor for reduction in stuttering frequency during DAF. Short delays reduced stuttering frequency by $87 \%$ at the fast speech rate and by $72 \%$ on average at the normal speech rate (Kalinowski et al., 1993). Delays of 50 and $75 \mathrm{~ms}$ were found to be most effective for stuttering reduction at both speech rates (Kalinowski et al., 1996).

There are individual differences in degree of reduction in stuttering with AAF (Armson \& Stuart, 1998; Hargrave, Kalinowski, Stuart, Armson, \& Jones, 1994; Natke, 2000; Stuart, Kalinowski, Armson, Stenstrom, \& Jones, 1996). The tendency of people to react differently to AAF exposure has been explained by degree of stuttering severity (Naylor, 1953; Ham \& Steer, 1967; Soderberg, 1969), by changes in frequency and complexity of the stuttering with age (Burke, 1975), and by maturation and learning (MacKay, 1968). Recent literature suggests that individual variability is a result of multi-causality of the disorder and the existence of subtypes of stuttering (Howell, Davis, \& Williams, 2006; Yairi, 2007). It has been suggested that different subtypes of stuttering respond differently to AAF exposure (Foundas et al., 2004). Individual differences could be influenced by the degree of dependency on auditory feedback for speech monitoring (Kent, 2000), by differences in auditory sensitivity (Howell et al., 2006; Howell \& Williams, 2004), or by individual abnormalities in structural and functional organisation of the auditory cortices (Braun et al., 1997; Foundas et al., 2004; Fox et al., 1996; Salemlin et al., 1998), or motor-and language-related brain areas (Watkins, Smith, Davis, \& Howell, 2008). 
There is no evidence that the effects of AAF carry over to situations where AAF is not present. Therefore, the clinical application of AAF seems to be limited to its use as a prosthetic device. According to the literature, excessive training is not required for effective use of AAF (Kalinowski, Dayalu, \& Saltuklaroglu, 2001). AAF is effective during both reading and spontaneous speech tasks, as well as in traditionally difficult situations such as using the telephone and speaking in front of audience (Armson et al., 2006; Kalinowski, Guntupalli, Stuart, \& Saltuklaroglu, 2004; Kalinowski, Saltuklaroglu, Guntupalli, \& Stuart, 2004). Because of the need to control variables, most researchers have investigated the effects of AAF on stuttering while participants read (Armson, Foote, Witt, Kalinowski, \& Stuart, 1997; Ham \& Steer, 1967; Kalinowski, Stuart, Wamsley, \& Rastatter, 1999; MacLeod et al., 1995; Maraist \& Hutton, 1957; Stuart, Kalinowski, \& Rastatter, 1997). Typically the effectiveness of AAF during reading tasks is assessed by measuring percentage of stuttered syllables (\%SS) while participants are reading a short passage. In general, the use of DAF and FAF leads to reductions in stuttering frequency by $50-90 \%$ during reading aloud. The most recent review of these studies was conducted by Lincoln et al. (2006). Reading is traditionally viewed as an easier activity for people who stutter than monologue speech production (Bloodstein, 1950; Venkatagiri, 2005). In real life, however, adults read aloud quite rarely in comparison to the amount of time they spend producing short monologues or taking part in spontaneous conversation. Additionally, the reduction in stuttering frequency that is observed during reading is not always apparent during spontaneous speech (Ingham, Moglia, Frank, Ingham, \& Cordes, 1997). Thus, it is important to test fluency techniques in spontaneous speech situations or monologue speech production. Monologue is easier to control in a research setting, although spontaneous conversation has more relevance to everyday life. Only a few studies have looked at the effect of the AAF on stuttering during spontaneous speech (Armson et al., 2006; Armson \& Stuart, 1998; Burke, 1975; Ingham et al., 1997; Stuart et al., 2004; Van Borsel et al., 2003; Zimmerman, Kalinowski, Stuart, $\&$ Rastatter, 1997). Careful examination of these studies reveals discrepancies in research findings.

The first study that addressed this issue was conducted in 1997 by Ingham et al. (1997). Comparisons of the effects of downward and upward one octave frequency shift on stuttering frequency were made during reading and spontaneous speech in comparison with a control condition. For the spontaneous speech task, the participants were asked to produce a short talk for $3 \mathrm{~min}$ on self-chosen topics. The researchers reported results for four people only. The results of the study and the application of the time-interval measures were criticized by Kalinowski, Stuart, and Armson (1997) as biased.

Zimmerman et al. (1997) investigated the effectiveness of DAF and FAF during scripted telephone conversations. Speaking on the telephone is one of the most difficult speech tasks for people who stutter. This study is usually cited as an example of evidence for the effectiveness of AAF during more natural situations or conversational speech (Howell, 2004; Onslow, 2001). Zimmerman et al. (1997) found a 55\% reduction in stuttering with FAF (downward $1 / 2$ octave frequency shift) and 60\% reduction in stuttering with DAF (50 ms delay). However, the effects of AAF were measured during scripted, rather than spontaneous, telephone conversations. It is not clear whether participants learned the scripts by heart, or read them, or read them repeatedly and then reproduced the scripts from memory.

The clinical significance of FAF use during monologue speech production was questioned by Armson and Stuart (1998). Upward and downward frequency shifts were tested with FAF parameters during reading and monologue and compared with a control condition. For the monologue task the researcher used cards with a general topic and a set of questions for each topic to maintain a smooth flow of monologue speech. The researchers reported on both individual and group data, emphasising the importance of both, as great variability across individuals is seen in previous research. Analysis of the group data showed that differences in stuttering frequency during reading were statistically significant for FAF versus the control condition. In contrast, stuttering frequency during monologue speech production task was not significantly altered by FAF. Substantial individual differences were identified. Summing up, the authors concluded that “. . .therapeutic application of a prosthetic device delivering FAF may be limited” (Armson \& Stuart, 1998, p. 487). Armson and Stuart concluded that greater frequency shifts may be needed and individuals may require different FAF parameters. Armson et al. (2006) investigated the effectiveness of AAF during reading, monologue speech production, and conversation tasks. On average, a greater reduction in stuttering frequency during monologue and conversation tasks (30\% and 36\%, respectively) was revealed than in the Armson and Stuart (1998) study. The AAF settings used by Armson et al. (2006) were selected individually for each participant. Individual selection of AAF parameters was also applied in two recent studies (Armson \& Kiefte, 2008; O’Donnel, Armson, \& Kiefte, 2008). Armson and Kiefte (2008) examined effectiveness of AAF during reading and monologue in 31 adults. On average, stuttering frequency reduction was $78.8 \%$ for reading and $60.7 \%$ for monologue. O'Donnel et al. (2008) used individual AAF parameters in the laboratory before and after their use in situations of every day living for 9-12 weeks in seven participants. 
On average, reduction in stuttering frequency during monologue speech production during the second laboratory assessment was $14 \%$ in comparison to $87 \%$ during the first laboratory assessment.

The effectiveness of AAF during monologue speech is not clear. Positive results that remain stable over time have been obtained in two studies only (Armson et al., 2006; Van Borsel et al., 2003). Previous research findings regarding the effectiveness of AAF for monologue speech production are inconclusive or controversial (Armson \& Stuart, 1998; Ingham et al., 1997; Zimmerman et al., 1997). Different equipment to deliver AAF and different parameters of AAF were applied in the studies. The present study investigated the effects of AAF produced by a commercially available portable anti-stuttering device, The Pocket Speech Lab (Casa Futura Technologies ${ }^{\circledR}$ ), during monologue speech production. Eight different parameters of AAF were compared with a control condition. Four types of combined DAF and FAF and two types of DAF were investigated. As stuttering has a tendency to fluctuate throughout different days or even across a day (Bloodstein, 1995), eight altered auditory feedback conditions and the control condition were tested twice during two separate consecutive testing sessions. The improvement in fluency was measured using percentage of stuttered syllables (\%SS) as the dependent variable. The present study addressed the following primary research questions:

1. What are the effects of eight different parameters of AAF on stuttering during monologue speech production?

2. Are there optimal AAF parameters for monologue speech production among the AAF experimental conditions tested?

Additionally, the relationships between effect of AAF and (a) the two types of speech task, reading and monologue speech production, and (b) the degree of stuttering severity were examined.

\section{Method}

\subsection{Participants}

The present study reports on eight native speakers of English (seven right-handed, one left-handed) aged 16-55 years old (mean 35 years, standard deviation 12.95). The participants, seven males and one female, were recruited from The University of Auckland Speech-language Therapy Clinic, the Stuttering Treatment and Research Trust (START) and via a self-help support group, Speak Easy, based in Auckland (New Zealand). The inclusion criteria for participation in the study were that the participants were:

1. not currently involved in any speech language therapy programme;

2. hearing sensitivity within normal limits $(25 \mathrm{~dB} H L$ or better at frequencies from 250 to $8000 \mathrm{~Hz}$ ) (American National Standards Institute, 1996); and

3. not diagnosed with any speech or language disorders other than developmental stuttering.

All participants had hearing thresholds of $15 \mathrm{~dB} \mathrm{HL}$ or better at $500-8000 \mathrm{~Hz}$. At $250 \mathrm{~Hz}$, five participants had hearing thresholds of $15 \mathrm{~dB}$ HL or better in both ears, and three had hearing thresholds of $15 \mathrm{~dB} \mathrm{HL}$ or better in one ear and $20 \mathrm{~dB}$ HL in the other ear. Thus, all participants had essentially normal hearing (Clark, 1981).

The degree of stuttering severity of the participants was measured using the Stuttering Severity Instrument for Children and Adults (SSI-3; Riley, 1994). This instrument includes assessment of stuttering severity during both reading and monologue production speech tasks. Based on this assessment, stuttering severity was "very mild" for one, "mild" for three, "moderate" for two and "severe" for two participant/s. Demographic data and scores on three stuttering measures derived from the Stuttering Severity Instrument for Children and Adults-Third Edition (SSI-3; Riley, 1994) and total overall scores are presented in Table 1.

\subsection{AAF apparatus and experimental conditions}

The Pocket Speech Lab is a portable device that was used to deliver AAF in the present study. The device was chosen because of its range of functions and comparatively low cost. The device does not require individual fitting with a personalised earpiece and hence is suitable for research purposes. The participants heard their own speech in both 
Table 1

Speech fluency profile obtained from the participants using the Stuttering Severity Instrument for Children and Adults (SSI-3; Riley, 1994). The severity category for the youngest participant (Participant 4) was determined using Children's Severity Conversion Table (Riley, 1994). The severity category for the adult participants was determined using Adults Severity Conversion Table (Riley, 1994). M: male, F: female, V/mild= very mild.

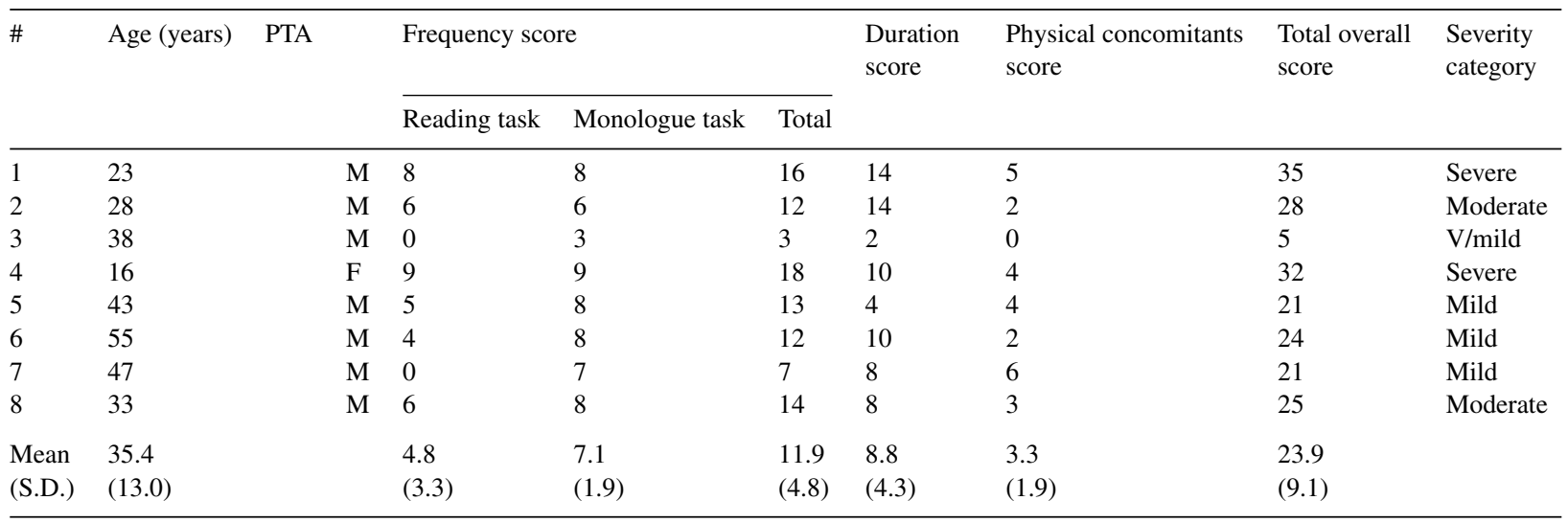

ears via a Sennheiser PC130 headset. The headset consists of headphones and a noise-cancelling boom microphone. The Pocket Speech Lab has controls that change frequency, intensity, and timing of auditory feedback.

Calibration of the Pocket Speech Lab frequency shift, time delay, and volume control was conducted in a doublewalled audiometric test suite. For the frequency shift calibration a $1000 \mathrm{~Hz}$ tone was generated by a clinical audiometer (Grason Stadler GSI 61) via a GSI loudspeaker. The microphone of the Sennheiser PC130 headset attached to the Pocket Speech Lab was placed on a stand, at $12 \mathrm{~cm}$ distance from the centre of the loudspeaker. The microphone output was fed to the Pocket Speech Lab where the signal frequency is shifted by the internal processor (Yamaha ${ }^{\circledR}$ YSS222 Karaoke Processor B). The Pocket Speech Lab outputs the frequency shifted signal to the headset. The headset earphone was placed on a $6 \mathrm{~cm}^{3}$ coupler (Brüel and Kjær Model 4152 with NBS 9A coupler) attached to a precision sound level meter (Brüel \& Kjær Type 2235). The output of the sound level meter was connected to a Standard Research Systems spectrum analyzer (SR770 FFT) from which the values of the frequency shift were obtained, by comparing the frequency spectrum of the Pocket Speech Lab output to the $1000 \mathrm{~Hz}$ frequency input signal. Because the frequency dial is not labelled, frequency shifts corresponding to divisions marked on the scale were determined empirically. Results of this calibration are presented in Table 2. To check for consistency the dial was shifted and measurements were repeated. For each dial setting, results were the same for the two measurements.

The same equipment was used to calibrate the intensity of the speech signals delivered by the earphones. The input level was measured adjacent to the headset microphone using a free field sound level meter (Brüel and Kjær Type 2235) with half inch microphone. The corresponding earphone output levels were measured in the $6 \mathrm{cc}$ coupler for different volume control settings. Increasing the volume setting from 2 to 5 produced an increase in gain from 0 to 11 decibels.

The time delay was calibrated by recording the input and output signals of the Pocket Speech Lab simultaneously using a two channel oscilloscope (Agilent $54622 \mathrm{~A}$ ). A short $1000 \mathrm{~Hz}$ tone, produced using Adobe ${ }^{\circledR}$ Audition $^{\mathrm{TM}} 1.5$ software and delivered via a computer sound card, was used as the input for this calibration. Measured time delays were $47.9 \mathrm{~ms}( \pm 0.5 \mathrm{~ms})$ and $75.4 \mathrm{~ms}( \pm 0.5 \mathrm{~ms})$ for the dial settings labelled " $55 \mathrm{~ms}$ " and "70 ms", respectively.

Table 2

Frequency shift calibration results for the Pocket Speech Lab.

\begin{tabular}{lllccr}
\hline $\begin{array}{l}\text { Frequency shift } \\
\text { dial setting }\end{array}$ & $\begin{array}{l}\text { Recorded } \\
\text { frequency }(\mathrm{Hz})\end{array}$ & $\begin{array}{l}\text { Target frequency } \\
(\mathrm{Hz})\end{array}$ & $\begin{array}{l}\text { Recorded \% shift } \\
\text { re: } 1000 \mathrm{~Hz}\end{array}$ & $\begin{array}{l}\text { Target \% shift re: } \\
1000 \mathrm{~Hz}\end{array}$ & $\begin{array}{l}\text { Approximate } \\
\text { octave shift }\end{array}$ \\
\hline $4 \frac{2}{3}$ & 1125.0 & 1148.7 & 12.5 & 14.9 & 0 \\
4 & 1007.8 & 1000.0 & 0.8 & -20.6 & 0 \\
$3 \frac{2}{3}$ & 804.69 & 793.7 & -19.5 & -29.3 & $-1 / 5$ \\
$3 \frac{1}{2}$ & 726.56 & 707.1 & -27.3 & $-1 / 2$ \\
\hline
\end{tabular}


Table 3

Experimental conditions tested and their actual values. (+) Upward frequency shift. (-) Downward frequency shift.

\begin{tabular}{|c|c|c|}
\hline Experimental condition code & Frequency shift actual value (octave) & Delay interval actual value (ms) \\
\hline 1 & 0 & 0 \\
\hline 2 & 0 & 50 \\
\hline 3 & $+1 / 5$ & 50 \\
\hline 4 & $-1 / 3$ & 50 \\
\hline 5 & $-1 / 2$ & 50 \\
\hline 6 & 0 & 75 \\
\hline 7 & $+1 / 5$ & 75 \\
\hline 8 & $-1 / 3$ & 75 \\
\hline 9 & $-1 / 2$ & 75 \\
\hline
\end{tabular}

The selection of the experimental conditions was based on previous research findings and the potential of the Pocket Speech Lab to deliver particular types of auditory feedback alteration. Calibration of the device showed that it is not possible to have a frequency shift feedback without also having a time delay. This influenced the choice of combined experimental conditions of frequency shifted and delayed auditory feedback. Algorithms of other commercial devices, e.g. SpeechEasy, can generate a frequency shift without a time delay. In previous research (Ingham et al., 1997) frequency shift of one octave was found to reduce speech naturalness and intelligibility. Therefore this frequency shift was excluded. A frequency shift of one-quarter octave was found optimal for reading but was not effective for monologue (Armson \& Stuart, 1998). These authors suggested that greater shifts in frequency may be more effective for monologue (Armson \& Stuart, 1998). To test this hypothesis two frequency shifts greater than and one less than one-quarter octave were used. The chosen values of DAF were based on evidence that 50 and $75 \mathrm{~ms}$ delay intervals are most effective during reading (Kalinowski et al., 1996). A control condition, with audio-feedback routed through the headphones non-altered, and eight conditions with feedback alterations were used. These are presented in Table 3 with their measured values.

\subsection{Procedure}

The study included three 90 min sessions: an introductory session and two testing sessions. Sessions were conducted in a quiet room at The University of Auckland Speech-language Therapy Clinic.

The first introductory session had five components:

1. initial interview to obtain demographic information,

2. hearing screening,

3. obtaining speech samples for stuttering severity assessment using The Stuttering Severity Instrument for Children and Adults (SSI-3; Riley, 1994),

4. selection of topics for monologue speech production for testing sessions,

5. familiarization with $\mathrm{AAF}$ device for $30 \mathrm{~min}$.

The Stuttering Severity Instrument for Children and Adults (SSI-3; Riley, 1994) includes assessment of stuttering severity during both reading and monologue production speech tasks. The participants were asked to read a 125 -word passage and to produce a short monologue about their job or school for $3 \mathrm{~min}$. They were instructed to talk at a comfortable speed and not to use any artificial strategy or technique to control or modify stuttering during speech production. For the reading task the phonetically balanced "Grandfather" passage (Darley, Aronson, \& Brown, 1975) was used.

For the two testing sessions investigating the effect of AAF on stuttering the participants were required to do nine monologues per session. To facilitate this, the participants chose their own topics during the introductory session. The eighteen general topics were chosen by each participant from a list of thirty suggested by the researcher. The goal was for the participants to be able to produce 3-min monologues with similar ease across conditions during two testing sessions. The participants were able to create their own topics or modify the suggested topics. All but two of the participants were happy to select topics from the list. Two participants chose two of their own topics to add to 16 
topics from the list. To prevent a rehearsal effect (Bloodstein, 1995) the participants were not given a copy of the list of topics they decided upon and were instructed not to plan or practise their speeches prior to the testing session. The list of general topics suggested by the researcher is attached as an Appendix to the manuscript. The topics were printed on separate cards that the participants picked up randomly during the testing sessions.

The final component of the introductory session was intended to familiarise the participant with the AAF device. The participants used the Pocket Speech Lab for $30 \mathrm{~min}$ with various parameters of AAF, and the main functions of the device were explained to them. The preferred level of feedback signal intensity was determined during this first session for each participant. To do that the participants were asked to manipulate the volume control of the device while reading a short passage from a magazine. The participants were instructed to choose a comfortable level that allowed them to hear themselves through the headphones but not through the air. All participants chose volume control settings of 2-3; these settings provided amplification of 5 decibels or less. The volume control setting was noted and the same setting was used in both testing sessions.

The two testing sessions were separated by at least six days with each participant. The average time between two testing sessions was 11 days $($ S.D. = 5.19). All nine experimental conditions (eight AAF conditions and the control condition) were tested in different random orders during the two testing sessions. Stuttering for each monologue was measured as \%SS using a speech rating instrument - the "True Talk" (Synergistic Electronics Pty. Ltd). The same examiner, the first author, conducted testing during these two testing sessions.

When the participants arrived for the testing sessions they were seated comfortably at a table. The Pocket Speech Lab and nine cards with the topics printed were already on the table. The cards were faced down, so the participants were not able to see the topic printed on them. The microphone used to record the speech samples was located on the table directly in front of the participants. The participants picked up one card with the topic. After 1-2 min, when the participants were ready to produce a monologue, the researcher placed the headset on and gave the instruction. For each condition the participants were instructed to talk for at least $3 \mathrm{~min}$ at a comfortable speed on the topic chosen by them. They were also told not to use any strategies and techniques that they used normally or had been taught previously to control or modify their stuttering. To establish continuous talking prior to each monologue, the participants were given 2 min to organise their thoughts and to make a plan for their talk. On average, approximately 1 min was required by them for this purpose. Occasionally, the researcher provided a verbal prompt to the participants in the form of a question to ensure a continuous flow of monologue speech production during the $3 \mathrm{~min}$ (Stuart et al., 2004).

To eliminate any possible carry over effect from the previous test condition the headset was removed at the end of the monologue and the participants were given sufficient time to recover from the previous setting of the device before experiencing a subsequent tested condition. For at least $5 \mathrm{~min}$ the participants talked with the researcher with the device switched off and the headset removed.

All experimental sessions were recorded on DVD + RW disks using a speed dome video camera and a Macrovision Corporation DVD Recorder.

\subsection{Dependent measures and reliability}

Speech samples from the DVD recordings were used for analysis of each test condition. The first 3 min of monologue speech produced by the participants were used for analyses. To ensure good intra- and inter-judge agreement the Lidcombe Behavioural Data Language (LBDL) was used to consistently identify stuttering moments. The LBDL has been described as a valid and reliable tool to characterize stuttering behaviour (Teesson, Packman, \& Onslow, 2003). On average, intra-judge and inter-judge agreement was $84.7 \%$ and $75 \%$, respectively, for experienced speech language pathologists working with people who stutter.

A syllable was classified as stuttered if it contained any of three types of speech mechanism activity: repeated movements, fixed postures or superfluous behaviours (Packman \& Onslow, 1998). To ensure accurate and consistent syllable counting the intraverbals such as "ah" or "um" were counted as independent minimal syllables (Roach, 2000). Excessive use of intraverbals, incomplete phrases or phrase revisions was classified as verbal superfluous behaviour. Inappropriate head or torso movements, facial grimaces as well as noisy breathing, whistling or clicking were defined as non-verbal superfluous behaviours in the current study. If the verbal superfluous behaviour moment was followed by the fixed posture this combination was counted as one moment of stuttering and was coded as a fixed posture.

To determine intra- and inter-judge reliability of $\%$ SS measures, intraclass correlations were calculated using version 12 of the Statistical Package for Social Science for Windows (SPSS). Fifteen (10\%) of the collected speech samples 
were randomly chosen and overall frequency counts of stuttering events was recalculated using the "True Talk" by the researcher to establish intra-judge reliability $(r=0.99)$. The independent researcher/clinician repeated the analysis of overall frequency counts of stuttering events for the same speech samples. Inter-judge agreement was very high $(r=0.91)$. Both judges were qualified speech-language pathologists with experience in identifying stuttering and in using LBDL and the "True Talk".

\subsection{Statistical analysis}

Statistical analysis was carried out using version 12 of the Statistical Package for Social Science for Windows (SPSS) and version 6.1 of Statistica (StatSoft, Inc.). A paired samples $t$-test or repeated measures analyses of variance were performed. To test if the variances of the differences between dependent variables were equal, Mauchly's Test of Sphericity was performed. When the assumption of sphericity was violated the Huynh-Feldt correction was used to adjust degrees of freedom and adjusted $p$ value was used to determine statistical significance. Where main effects were found, planned comparisons were performed between the control condition and the altered auditory feedback conditions to determine which differences were statistically significant. The significance level was set at 0.05 for all statistical tests. Bonferroni adjustments were made to correct for multiple comparisons.

\section{Results}

\subsection{Degree of difficulty of reading versus monologue speech production}

During the severity testing, stuttering frequency was measured in percentage of words stuttered (\%WS) (Riley, 1994) and in percentage of syllables stuttered (\%SS) for both reading and monologue speech tasks. Stuttering frequency during reading and monologue speech production tasks measured in \%SS was analysed to examine the effect of AAF on stuttering frequency during two speech tasks. Only Participant 4 demonstrated more stuttering in reading than in monologue whereas seven participants (87.5\%) stuttered more during monologue speech production. On average, stuttering was about 3-4\% more frequent during monologue speech production. Table 4 presents the individual raw data and the mean stuttering frequency in $\%$ SS during reading and monologue speech production. To compare the average stuttering frequency (\%SS) during reading and monologue tasks, a paired $t$-test analysis was performed using a two-tailed distribution. The paired $t$-test results supported the null hypothesis that the type of speech task has no significant effect on stuttering frequency $[t=1.77$; d.f. $=7 ; p=0.119]$.

\subsection{Effect of altered auditory feedback on stuttering frequency - individual data}

The effect of altered auditory feedback on fluency was determined using percentage syllables stuttered as the dependent variable. Two participants $(25 \%)$ demonstrated stuttering reduction under all altered auditory feedback experimental conditions compared to the control condition. All participants showed a consistent change across the two sessions for at least one AAF condition. The degree of stuttering reduction varied across the experimental conditions.

Table 4

Stuttering frequency during reading and monologue speech production. $\% \mathrm{SS}=$ percentage of syllables stuttered; S.D. = standard deviation.

\begin{tabular}{lcr}
\hline Participant number & Reading \%SS & Monologue \%SS \\
\hline 1 & 9.6 & 15.4 \\
2 & 5.4 & 6.5 \\
3 & 0.6 & 1.4 \\
4 & 29.8 & 21.4 \\
5 & 2.4 & 8.2 \\
6 & 1.8 & 10.0 \\
7 & 0.0 & 5.9 \\
8 & 4.9 & 14.4 \\
Mean & 6.8 & 10.4 \\
S.D. & 9.8 & 6.4 \\
\hline
\end{tabular}


Table 5

Individual data on maximal and minimal stuttering reduction and percentage of reduction in stuttering frequency during the first testing session. $\% \mathrm{SS}=$ percentage of syllables stuttered. A negative reduction in stuttering frequency value indicates that stuttering frequency was higher for this experimental condition than for the control condition. S.D. = standard deviation. The percentage of reduction in stuttering was calculated using formula: $\left(\% \mathrm{SS}_{\text {control condition }}-\% \mathrm{SS}_{\text {experimental condition }}\right) \times 100 / \% \mathrm{SS}_{\text {control condition }}$.

\begin{tabular}{|c|c|c|c|c|c|c|c|}
\hline \multirow[t]{3}{*}{ Participant number } & \multicolumn{7}{|l|}{ Testing session 1} \\
\hline & \multirow[t]{2}{*}{ Control condition $\% \mathrm{SS}$} & \multicolumn{3}{|c|}{ Maximal stuttering reduction } & \multicolumn{3}{|c|}{ Minimal stuttering reduction } \\
\hline & & $\begin{array}{l}\text { Experimental } \\
\text { condition }\end{array}$ & $\% \mathrm{SS}$ & $\begin{array}{l}\text { Reduction in } \\
\text { stuttering \% }\end{array}$ & $\begin{array}{l}\text { Experimental } \\
\text { condition }\end{array}$ & $\% \mathrm{SS}$ & $\begin{array}{l}\text { Reduction in } \\
\text { stuttering \% }\end{array}$ \\
\hline 1 & 20.7 & 3,5 & 1.4 & 93 & 6 & 9.4 & 55 \\
\hline 2 & 6.5 & 6 & 3.0 & 54 & 4 & 11.1 & -71 \\
\hline 3 & 1.1 & 4 & 0.1 & 91 & 5 & 2.0 & -82 \\
\hline 4 & 19.1 & 9 & 14.7 & 23 & 2 & 22.5 & -18 \\
\hline 5 & 6.9 & 5 & 0.2 & 97 & 4,8 & 5.5 & 20 \\
\hline 6 & 15.2 & 9 & 5.3 & 65 & 8 & 14.0 & 8 \\
\hline 7 & 3.8 & 9 & 1.3 & 66 & 2 & 7.8 & -105 \\
\hline 8 & 13.0 & 4 & 3.2 & 75 & 5 & 7.3 & 44 \\
\hline Mean & 10.8 & & 3.7 & $71 \%$ & & 10.0 & $-19 \%$ \\
\hline S.D. & 7.3 & & 4.8 & $25 \%$ & & 6.2 & $61 \%$ \\
\hline
\end{tabular}

Six participants $(75 \%)$ demonstrated mixed results across testing sessions for at least one experimental condition. To determine the direction and extent of changes in stuttering frequency produced by altered auditory feedback, maximal and minimal stuttering reductions were determined and compared with the control condition for each participant for each testing session. Individual data on experimental conditions producing maximal (i.e., most fluent) and minimal (i.e., least fluent) stuttering reduction fluency levels and percentage of stuttering frequency reduction are presented in Tables 5 and 6.

Tables 5 and 6 show that half of the participants stuttered more often in the experimental condition that produced the minimal reduction in stuttering frequency than in the control condition (negative stuttering reduction value). Only Participants 2, 4 and 5 demonstrated consistency in the AAF condition that produced the maximal stuttering reduction over the two testing sessions. Only Participants 5 and 8 (25\% of participants) demonstrated consistency in the AAF condition that produced the minimal stuttering reduction over the two testing sessions. The reduction of stuttering frequency varied across individuals from 23 to $97 \%$ during the first testing session and from 3 to $88 \%$ during the

Table 6

Individual data on maximal and minimal stuttering reduction and percentage of reduction in stuttering frequency during the second testing session. $\% \mathrm{SS}=$ percentage of syllables stuttered. A negative reduction in stuttering frequency value indicates that stuttering frequency was higher for this experimental condition than for the control condition. S.D. = standard deviation. The percentage of reduction in stuttering was calculated using formula: $\left(\% \mathrm{SS}_{\text {control condition }}-\% \mathrm{SS}_{\text {experimental condition }}\right) \times 100 / \% \mathrm{SS}_{\text {control condition }}$.

\begin{tabular}{|c|c|c|c|c|c|c|c|}
\hline \multirow[t]{3}{*}{ Participant number } & \multicolumn{7}{|l|}{ Testing session 2} \\
\hline & \multirow[t]{2}{*}{ Control condition $\% \mathrm{SS}$} & \multicolumn{3}{|c|}{ Maximal stuttering reduction } & \multicolumn{3}{|c|}{ Minimal stuttering reduction } \\
\hline & & $\begin{array}{l}\text { Experimental } \\
\text { condition }\end{array}$ & $\% \mathrm{SS}$ & $\begin{array}{l}\text { Reduction in } \\
\text { stuttering \% }\end{array}$ & $\begin{array}{l}\text { Experimental } \\
\text { condition }\end{array}$ & $\% \mathrm{SS}$ & $\begin{array}{l}\text { Reduction in } \\
\text { stuttering \% }\end{array}$ \\
\hline 1 & 19.6 & 8 & 3.6 & 82 & 2 & 16.9 & 14 \\
\hline 2 & 7.0 & 6 & 6.8 & 3 & 7 & 12.8 & -83 \\
\hline 3 & 1.0 & 8 & 0.5 & 50 & 3 & 1.2 & -20 \\
\hline 4 & 19.5 & 9 & 14.7 & 25 & 8 & 26.7 & -37 \\
\hline 5 & 2.1 & 5 & 1.0 & 52 & 8 & 2.4 & -14 \\
\hline 6 & 10.0 & 5 & 6.5 & 35 & 2 & 14.2 & -42 \\
\hline 7 & 5.4 & 4 & 2.1 & 61 & 9 & 4.7 & 13 \\
\hline 8 & 12.8 & 7 & 1.5 & 88 & 5 & 5.1 & 60 \\
\hline Mean & 9.7 & & 4.6 & $50 \%$ & & 10.5 & $-14 \%$ \\
\hline S.D. & 7.2 & & 4.7 & $28 \%$ & & 8.8 & $43 \%$ \\
\hline
\end{tabular}




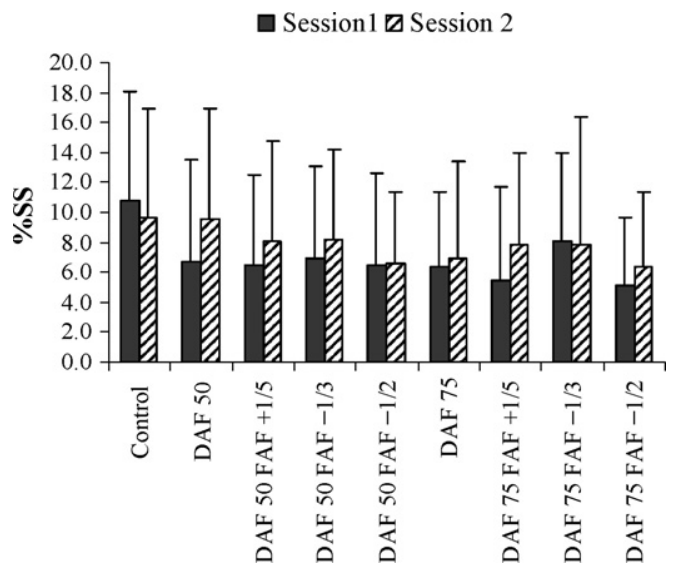

Fig. 1. Mean percentage of syllables stuttered ( $N=8$ participants) for the nine experimental conditions (control and eight altered auditory feedback conditions) during the two testing sessions. Error bars show standard deviations. DAF: delayed auditory feedback, FAF: frequency shifted auditory feedback, \%SS: percentage of syllables stuttered.

second one. Six participants demonstrated a decline in the AAF stuttering reduction effect during the second session to various degrees. Only Participants 4 and 8 showed an increase in percent of stuttering reduction, from 75 to $88 \%$ and from 23 to $25 \%$, respectively, over the two testing sessions. This maximal stuttering reduction was for different experimental conditions, however, for Participant 8.

\subsection{Effect of altered auditory feedback on stuttering frequency - group data}

There were two testing sessions and nine experimental conditions (control plus eight altered auditory feedback conditions). Fig. 1 illustrates the mean percentage of syllables stuttered produced for each AAF experimental condition versus the control condition during the two testing sessions. The effects of the experimental condition and test session on the participants' stuttering frequencies $(\% \mathrm{SS})$ were investigated using two-way repeated measures analysis of variance. The Mauchly's Sphericity Test was significant $\left[\chi^{2}=54.39\right.$; d.f. $\left.=35 ; p=0.019\right]$, therefore the Huynh-Feldt correction for degrees of freedom was used. This analysis showed a significant main effect of experimental condition $[F=2.89$; adjusted d.f. $=3.5,24.4$; adjusted $p=0.049]$. To further investigate the effect of experimental condition on stuttering frequency, planned comparisons with Bonferroni adjustment were performed between the control condition and each altered auditory feedback condition. Planned comparisons revealed that Experimental Condition 6 (DAF $75 \mathrm{~ms}$ ) differed significantly from the control condition $[p=0.039]$. The difference between the control condition and Experimental Condition 9 (DAF $75 \mathrm{msec}$ FAF - 1/2 octave) approached statistical significance [ $p=0.052]$.

The main effect of session and the interaction between session and condition were not statistically significant. Although stuttering frequency did not change significantly between sessions, Fig. 1 shows that percentage syllables stuttered was slightly higher for seven of the eight altered auditory feedback experimental conditions during session two. The effect of Experimental Condition 5 (DAF $50 \mathrm{~ms}$ FAF - 1/2 octave) and Experimental Condition 8 (DAF $75 \mathrm{~ms}$ FAF $-1 / 3$ octave) on stuttering frequency was very stable across the testing sessions.

\subsection{Was the effect of the experimental condition influenced by severity of stuttering?}

To investigate the influence of the stuttering severity degree on the stuttering reduction effect of the AAF experimental conditions, the two-way repeated measures analysis of variance was repeated with total severity score as a covariate. The total severity score was obtained using the Stuttering Severity Instrument for Children and Adults (SSI-3; Riley, 1994). There were no statistical interactions between testing session and experimental condition, testing session and total severity score, or experimental condition and total severity score.

Individual data on the percentage of stuttering reduction for both Experimental Condition 6 (DAF $75 \mathrm{~ms}$ ) and Experimental Condition 9 (DAF $75 \mathrm{~ms}$ FAF - 1/2 octave) averaged across the two testing sessions were analysed further to investigate the effects of stuttering severity. Fig. 2 shows the individual percentage of stuttering reduction 


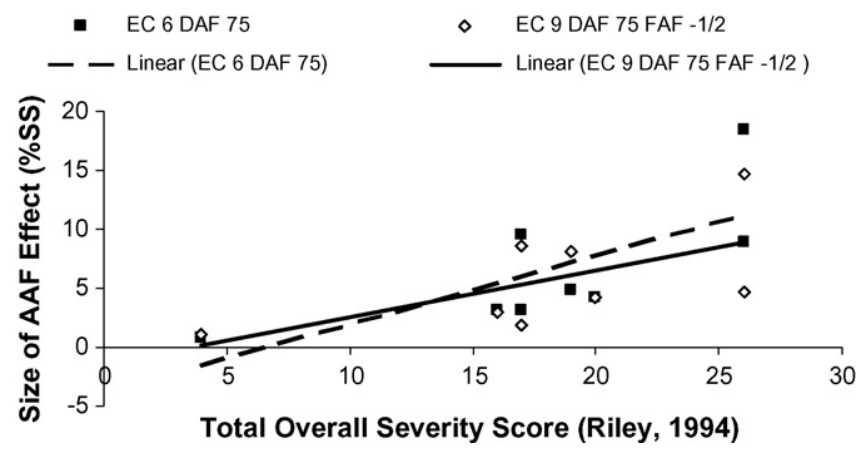

Fig. 2. Size of the stuttering reduction effect for individual participants ( $N=8$ participants) for Experimental Conditions 6 and 9 as a function of severity of stuttering. The size of the experimental condition positive effect on the stuttering frequency for each participant was calculated using the

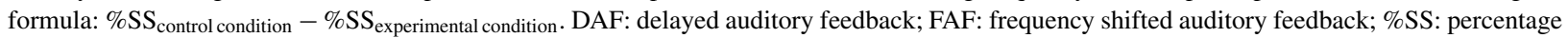
of syllables stuttered. The dashed line shows the linear regression line for Experimental Condition 6 (DAF $75 \mathrm{~ms}$ ) data. The solid line shows the linear regression line for Experimental Condition 9 (DAF 75 ms FAF - 1/2 octave) data.

and size of the reduction in stuttering effects for Experimental Conditions 6 and 9. Fig. 2 shows that, on average, the stuttering reduction produced by AAF Experimental Conditions 6 and 9 are equal. The standard deviation is much larger, however, for Experimental Condition 9 and for two participants this experimental condition had either no effect or negative effect on their stuttering frequency. The trend lines in Fig. 2 show, overall, that the more severe the stuttering, the greater the stuttering reduction effect produced by both conditions. There is no difference between the size of stuttering reduction for Experimental Conditions 6 and 9 for the participants with mild stuttering. For people with moderate stuttering the difference between the stuttering reductions with these two AAF experimental conditions becomes more remarkable, and there are major variations for people who stutter severely.

\subsection{Which experimental condition is optimal for monologue speech production?}

Tables 5 and 6 show that, for three participants, Experimental Condition 9 (DAF 75 ms FAF - 1/2 octave) produced the maximal reduction in stuttering frequency during the first testing session and this effect remained stable for one of these participants during the second testing session. To show the best result an experimental condition should produce maximal stuttering reduction as many times as possible, and at the same time produce the minimal reduction in stuttering frequency as few times as possible. Condition 6 (DAF $75 \mathrm{~ms}$ ) produced the maximal stuttering reduction for Participant 2 only, and this effect was stable across two sessions.

The number of times when each AAF conditions produced maximal and minimal reduction in stuttering frequency for both sessions for individual participants is presented in Table 7. If an experimental condition produced a consistent maximal (or minimal) stuttering reduction effect for the two testing sessions for the eight participants the number of cases would be 16. By contrast, if an experimental condition did not produce maximal (or minimal) stuttering reduction effect for the two testing sessions the number of cases would be nil. The best result for the experimental conditions

Table 7

Number of cases when the experimental conditions produced maximal and minimal reduction in stuttering frequency and the significance level when compared to the control condition.

\begin{tabular}{lllll}
\hline $\begin{array}{l}\text { Experimental } \\
\text { condition }\end{array}$ & $\begin{array}{l}\text { Type of auditory } \\
\text { feedback alteration }\end{array}$ & $\begin{array}{l}\text { Maximal } \\
\text { reduction }\end{array}$ & $\begin{array}{l}\text { Minimal } \\
\text { reduction }\end{array}$ & $\begin{array}{l}p \text { value for significance of difference } \\
\text { from the control condition }\end{array}$ \\
\hline 2 & DAF 50 & 0 & 4 & 0.141 \\
3 & DAF 50 FAF +1/5 & 1 & 1 & 0.141 \\
4 & DAF 50 FAF -1/3 & 3 & 2 & 0.082 \\
5 & DAF 50 FAF -1/2 & 4 & 3 & 0.074 \\
6 & DAF 75 & 2 & 1 & 0.039 \\
7 & DAF 75 FAF +1/2 & 1 & 1 & 0.075 \\
8 & DAF 75 FAF -1/3 & 2 & 4 & 0.346 \\
9 & DAF 75 FAF -1/2 & 4 & 1 & 0.051 \\
\hline
\end{tabular}


that produced maximal stuttering reduction was four. This result was obtained for Experimental Conditions 5 (DAF $50 \mathrm{FAF}-1 / 2$ octave) and 9 (DAF $75 \mathrm{FAF}-1 / 2$ ). In general, the experimental condition producing maximal reduction in stuttering frequency was not consistent across the participants or testing sessions.

\section{Discussion}

Overall, the results of the present study have confirmed that AAF is an effective means to reduce stuttering frequency during monologue speech production. On average, the maximal stuttering reduction was $71 \%$ for the first testing session and 50\% for the second testing session. Maximal stuttering reductions were not achieved with the same experimental condition across participants, however. An important finding of the study is that the stuttering reduction effect was not equal across experimental conditions. All eight AAF experimental conditions in the present study reduced stuttering frequency to varying degrees. However, only Experimental Condition 6 (DAF 75 ms) produced a reduction of stuttering frequency that reached statistical significance $[p<0.05]$. On average, the stuttering reduction produced by Experimental Condition 6 (DAF $75 \mathrm{~ms}$ ) was 35\%. The effect of Experimental Condition 9 (DAF $75 \mathrm{~ms}$ FAF - 1/2 octave) on stuttering frequency approached statistical significance $[p=0.052]$. This condition produced $44 \%$ of stuttering reduction, on average. In comparison, $67 \%$ stuttering reduction during monologue speech production was obtained by Stuart et al. (2004), 30\% by Armson et al. (2006), and 60.7\% by Armson and Kiefte (2008). In these studies a SpeechEasy device was used to alter auditory feedback. The impact of technological differences between the SpeechEasy and the Pocket Speech Lab on their effectiveness is unknown. To the best of our knowledge, only one case study has been conducted comparing the impact of the SpeechEasy and the Pocket Speech Lab devices on stuttering frequency during reading in quiet and noisy experimental conditions (Hyde, 2003). Both devices appeared to be effective. The Pocket Speech Lab provided the greater percentage of fluent $3 \mathrm{~s}$ intervals. Different settings of the devices for both delay and frequency shift were used in the study, however, making it difficult to interpret these findings.

The amount of time delay appeared to be a critical factor in determining the degree of the stuttering frequency improvement. Both Experimental Condition 6 (DAF 75 ms) and Experimental Condition 9 (DAF 75 ms FAF -1/2 octave) included $75 \mathrm{~ms}$ delay. Experimental conditions with $50 \mathrm{~ms}$ delay produced less reduction in stuttering, and did not differ significantly from the control condition.

The importance of using longer time delays for monologue speech production than for reading to achieve significant reduction in stuttering frequency has been demonstrated in previous research. Van Borsel et al. (2003) and Andrews, Howie, Dozsa, and Guitar (1982) applied quite long time delays in their research and found DAF to be effective for monologue speech production. Van Borsel et al. (2003) used $93 \mathrm{~ms}$ delay and Andrews et al. used $250 \mathrm{~ms}$ delay. Time delays used by Andrews et al. (1982) and Van Borsel et al. (2003) are longer than the delays used in the present study. Long time delays of DAF typically associated with a noticeable reduction in speech rate, and therefore are not comparable to the effects of short delay DAF. Another disadvantage of the use of DAF, especially long time delays, is a decrease in perceived speech naturalness (Stuart \& Kalinowski, 2004). A shorter delay, similar to the present study, was used by Stuart et al. (2004), who used a combination of $60 \mathrm{~ms}$ delay and $500 \mathrm{~Hz}$ frequency shift and found an immediate reduction in stuttering of $67 \%$. Studies that applied FAF only have failed to improve fluency during monologue speech production (Armson \& Stuart, 1998; Ingham et al., 1997). Interestingly, in the current study it was a downward frequency shift combined with DAF that was more effective than a combination of the same DAF with an upward frequency shift. Stuart et al. (2004) did not compare an upward frequency shift with the downward frequency shift, but found that an upward frequency shift combined with DAF was effective. This difference in research findings could be explained by the fact that Stuart et al. (2004) followed the "SpeechEasy protocol" that includes the use of some motor techniques, whereas in the present study participants were instructed to not use any motor strategies.

The current study showed that not every combination of FAF and DAF tested produced significant stuttering reduction. The results indicate that a particular combination of FAF and DAF was key in achieving significant fluency enhancement, for combined experimental conditions. Unfortunately the device used in the present study limited the number of possible FAF and DAF setting, and FAF alone could not be investigated.

The stuttering reduction effects of AAF experimental conditions were quite stable across the two testing sessions. Although stuttering frequency was higher during the second session for six of the eight AAF experimental conditions, statistically significant differences in stuttering frequency were not found across the two testing sessions. However, the analysis of the individual data showed that there was no stability in the effects of the AAF experimental conditions that produced the maximal and the minimal fluency levels across the two testing sessions for individual participants. 
This variability reduces the ability to draw statistical conclusions about the impact of AAF. Larger sample sizes are recommended for future studies to determine what subject variables may be contributing to this variability. Only two participants achieved their maximal fluency levels for the same AAF experimental condition across testing sessions. This finding can be considered a warning regarding clinical use of AAF. Choice of optimal AAF parameters for the long term is important for clinical use of AAF in stuttering management. It is difficult to "prescribe" one AAF parameter as optimal for a client, if different AAF parameters achieve maximal fluency levels on different days. To the best of our knowledge, no studies conducted so far, excluding the present, have investigated the effect of several AAF parameters across testing occasions to determine the stability of stuttering reduction effects across AAF parameters. Experimental Condition 9 (DAF 75 FAF - 1/2 octave) produced the maximal fluency level more often than other AAF experimental conditions, four times (25\%) out of 16 maximal possible occasions (eight participants, two testing sessions). Experimental Condition 9 produced the minimal fluency level less often than other AAF experimental conditions. This condition also showed a difference from the control condition that approached statistical significance when the group data were analysed. Thus, this condition appeared to be the most effective overall. As there was some instability in the experimental conditions that produced the maximal fluency level across the two testing sessions, however, clinicians may have to select AAF parameters carefully for each client and review the effectiveness of AAF over time. The causes of this variability are not understood. With advances in neuroimaging leading to greater understanding of the nature of stuttering it may be possible to explain this variability better in the future.

Examination of the individual data showed that people with differing severity of stuttering reacted differently to AAF exposure. In general, stuttering frequency of people with more severe stuttering was reduced more than stuttering frequency of people with mild stuttering. This is consistent with the results of studies investigating the effect of DAF on stuttering during reading (Burke, 1975; Soderberg, 1969; Van Riper, 1971). Also, it was found that the degree of the reduction in stuttering frequency of people with severe stuttering is more variable across AAF parameters than for people with moderate stuttering. The greater stuttering reduction effect and greater variation for people with severe and moderate stuttering than for those with mild stuttering may be explained by a 'floor effect' for people with mild stuttering. This finding might be helpful for clinical practice because it might serve as a basis for clinical decision making regarding AAF parameter selection.

Comparison of stuttering frequency during reading and monologue speech production showed no significant difference in mean stuttering frequency. This is consistent with observations made by Armson and Stuart (1998) and Young (1980), but in conflict with the traditional assumption that stuttering frequency is greater during monologue speech production than during reading for people who stutter (Armson \& Stuart, 1998). Individual data showed that stuttering frequency during monologue speech production was less than during reading for seven of the eight participants. Only one participant demonstrated greater stuttering frequency during reading. The increase in stuttering frequency during monologue speech production for this one case was not due to reading difficulties.

\subsection{Conclusions and recommendations}

This study provides evidence that AAF reduces overt stuttering manifestations in adults who stutter during monologue speech production. The findings of previous research are inconsistent, as are the AAF parameters applied for monologue speech production. This was the first study, to the best of our knowledge, which systematically tested several AAF parameters twice. On average, a $75 \mathrm{~ms}$ time delay on its own and a combination of the $75 \mathrm{~ms}$ time delay and a half octave downward frequency shift were found to be more effective than other combinations of AAF parameters that were investigated. A $75 \mathrm{~ms}$ time delay produced an average reduction in stuttering frequency of $35 \%$. The combination of $75 \mathrm{~ms}$ time delay and a half an octave downward frequency shift produced, on average, $44 \%$ reduction in dysfluencies. The current research findings show individual variability in stuttering reduction across participants, as seen in previous experiments (Armson \& Kiefte, 2008; Armson et al., 2006; Armson \& Stuart, 1998; Ingham et al., 1997; Zimmerman et al., 1997). There was also some instability in the AAF parameters that produced maximal stuttering reduction across the two testing sessions. Thus, in clinical practice careful individual selection of AAF parameters and re-evaluation of the effect of AAF over time is recommended.

Additional research is needed to verify the current research findings of instability of AAF parameters producing maximal fluency levels across testing occasions. It is worth comparing the stability of the effect of pure FAF and DAF on stuttering frequency, as the device used in the present study did not have a pure FAF setting. Also, long term and well controlled studies are required to investigate the effectiveness of using the optimal AAF parameter for 
monologue speech production in isolation and in combination with traditional fluency shaping techniques. Comparisons of experimental and control groups are preferable for determining the role of AAF in fluency enhancement. Larger numbers of participants are also recommended, particularly since AAF effectiveness appears to depend on stuttering severity. There is also a need to investigate how fluency enhanced by AAF will be maintained and transferred into real-life situations.

\section{QUESTIONS}

1. The present study on AAF during monologue speech production is motivated by:

a. the lack of sufficient data from previous research

b. the mechanism of fluency improvement by AAF is not completely understood

c. the presence of discrepancies in previous research findings on the use of AAF during monologue speech production

2. Which one of the following is not a possible auditory feedback alteration performed by the "Pocket Speech Lab" (the device used in this study)

a. delayed auditory feedback

b. frequency shifted auditory feedback

c. a combination of delayed auditory feedback and frequency shifted auditory feedback

d. masked auditory feedback

3. In the present study, on average, the most marked reduction in stuttering frequency was demonstrated by the experimental conditions with a time delay of
a. $75 \mathrm{~ms}$
b. $50 \mathrm{~ms}$
c. none of the above
d. all of the above

4. Select the statement/s that best describe the overall results of the current study
a. all participants experienced a dramatic reduction in stuttering frequency for all tested experimental conditions;
b. the majority of participants experienced a reduction in stuttering frequency for at least one tested experimental condition;
c. the participants experienced no or minimal reduction in stuttering frequency for any of tested experimental conditions;
d. the participants experienced a wide range of responses to auditory alteration exposure

5. In this study, speech samples were obtained for each tested experimental conditions on two occasions. Analysis of individual data showed that:
a. all participants showed a consistent change across the two sessions for at least one AAF condition
b. two participants demonstrated consistency in the AAF condition that produced the minimal fluency level over the two testing sessions
c. all of the above
d. none of the above

\section{CONTINUING EDUCATION}

\section{Effects of altered auditory feedback (AAF) on stuttering frequency during monologue speech production}

\section{References}

Adams, M. R., Lewis, J. I., \& Besozzi, T. E. (1973). The effects of reduced reading rate on stuttering frequency. Journal of Speech and Hearing Research, 16, 671-675.

Andrews, G., Howie, P., Dozsa, M., \& Guitar, B. (1982). Stuttering: Speech pattern characteristics under fluency-inducing conditions. Journal of Speech and Hearing Research, 25, 208-216.

Armson, J., Foote, S., Witt, C., Kalinowski, J., \& Stuart, A. (1997). Effect of frequency altered feedback and audience size on stuttering. European Journal of Disorders of Communication, 32, 359-366. 
Armson, J., \& Kiefte, M. (2008). The effect of SpeechEasy on stuttering frequency, speech rate, and speech naturalness. Journal of Fluency Disorders, $33,120-134$.

Armson, J., Kiefte, M., Mason, J., \& De Croos, D. (2006). The effect of SpeechEasy on stuttering frequency in laboratory conditions. Journal of Fluency Disorders, 31, 137-152.

Armson, J., \& Stuart, A. (1998). Effect of extended exposure to frequency-altered feedback on stuttering during reading and monologue. Journal of Speech Language and Hearing Research, 41, 479-490.

Bloodstein, O. (1950). A rating scale study of conditions under which stuttering is reduced or absent. Journal of Speech and Hearing Disorders, 15, 29-36.

Bloodstein, O. (1995). A handbook on stuttering (5th ed.). San Diego: Singular Publishing Group, Inc.

Braun, A. R., Varga, M., Stager, S., Schulz, G., Selbie, S., \& Maisog, J. M. (1997). Altered patterns of cerebral activity during speech and language production in developmental stuttering. An H2 (15)O positron emission tomography study. Brain, 120, 761-784.

Burke, B. D. (1975). Variables affecting stuttering initial reactions to delayed auditory feedback. Journal of Communication Disorders, 8, 141155.

Clark, J. G. (1981). Uses and abuses of hearing loss classification. ASHA, 23, 493-500.

Costello-Ingham, J. C. (1993). Current status of stuttering and behavior modification-1. Recent trends in the application of behavior application in children and adults. Journal of Fluency Disorders, 18, 27-44.

Darley, F. L., Aronson, A. E., \& Brown, J. R. (1975). Motor speech disorders. Philadelphia, London, Toronto: W.B. Saunders Company.

Fairbanks, G. (1955). Selected vocal effects of delayed auditory feedback. Journal of Speech and Hearing Disorders, 20(4), 333-346.

Foundas, A. L., Bollich, A. M., Feldman, J., Corey, D. M., Hurley, M., Lemen, L. C., et al. (2004). Aberrant auditory processing and atypical planum temporale in developmental stuttering. Neurology, 63, 1640-1646.

Fox, P. T., Ingham, R. J., Ingham, J. C., Hirsch, T. B., Downs, J. H., \& Martin, C. (1996). A PET study of the neural systems of stuttering. Nature, $382,158-161$.

Goldiamond, I. (1965). Stuttering and fluency as manipulatable operant response classes. In L. Krasner \& L. P. Ulmann (Eds.), Research in behavior modification: New developments and implications. New York: Holt, Rinehart \& Winston.

Ham, R., \& Steer, M. D. (1967). Certain effects of alterations in auditory feedback. Folia Phoniatrica, 19, 53-62.

Hargrave, S., Kalinowski, J., Stuart, A., Armson, J., \& Jones, K. (1994). Effects of frequency-altered feedback on stuttering frequency at normal and fast speech rates. Journal of Speech and Hearing Research, 37, 1313-1319.

Howell, P. (2004). Effects of delayed auditory feedback and frequency-shifted feedback on speech control and some potentials for future development of prosthetic aids for stammering. Stammering Research, 1(1), 31-46.

Howell, P., Davis, S., \& Williams, S. M. (2006). Auditory abilities of speakers who persisted, or recovered, from stuttering. Journal of Fluency Disorders, 3, 257-270.

Howell, P., El-Yaniv, N., \& Powell, D. J. (1987). Factors affecting fluency in stutterers when speaking under altered auditory feedback. In H. F. M. Peters \& W. Hulstijn (Eds.), Speech motor dynamics in stuttering (pp. 361-369). New York: Springer Press.

Howell, P., \& Sackin, S. (2002). Timing interference to speech in altered listening conditions. Journal of the Acoustical Society of America, 111(6), $2842-2852$.

Howell, P., \& Williams, S. (2004). Development of auditory sensitivity in children who stutter and fluent children. Ear and Hearing, 25, 1-10.

Hyde, L. (2003). Comparison of the Speech Easy and Casa Futura/Jabra fluency devices. Presentation to the Canadian Association of Persons who Stutter conference. Accessed November 2004. Available at URL address: http://www.casafuturacanada.com/CF\%20SE\%20Study2.htm

Ingham, R. J., Moglia, R. A., Frank, P., Ingham, J. C., \& Cordes, A. K. (1997). Experimental investigation of the effect of frequency-altered auditory feedback on the speech of adults who stutter. Journal of Speech Language and Hearing Research, 40, 361-372.

Kalinowski, J., Armson, J., Mieszkowski, M. R., Stuart, A., \& Gracco, V. L. (1993). Effects of alterations in auditory feedback and speech rate on stuttering frequency. Language and Speech, 36(1), 1-16.

Kalinowski, J., Armson, J., \& Stuart, A. (1995). Effect of normal and fast articulatory rates on stuttering frequency. Journal of Fluency Disorders, 20, 293-302.

Kalinowski, J., Guntupalli, V., Stuart, A., \& Saltuklaroglu, T. (2004). Self-reported efficacy of an ear-level prosthetic device that delivers altered auditory feedback for the management of stuttering. International Journal of Rehabilitation Research, 27, 167170.

Kalinowski, J., Saltuklaroglu, T., Guntupalli, V., \& Stuart, A. (2004). Gestural recovery and the role of forward and reversed syllabic repetitions as stuttering inhibitors in adults. Neuroscience Letters, 363, 144-149.

Kalinowski, J., Stuart, A., \& Armson, J. (1997). Response to Ingham: Seeking the truthfulness of stuttering research data. Journal of Fluency Disorders, 22, 57-59.

Kalinowski, J., Stuart, A., Sark, S., \& Armson, J. (1996). Stuttering amelioration at various auditory feedback delays and speech rates. European Journal of Disorders of Communication, 31, 259-269.

Kalinowski, J., Stuart, A., Wamsley, L., \& Rastatter, M. P. (1999). Effects of monitoring condition and frequency-altered feedback on stuttering frequency. Journal of Speech Language and Hearing Research, 42, 1347-1354.

Kent, R. D. (2000). Research on speech motor control and its disorders: A review and prospective. Journal of Communication Disorders, 33, 391-428.

Lee, B. S. (1950). Effects of delayed speech feedback. Journal of the Acoustical Society of America, 22(6), 824-826.

Lincoln, M., Packman, A., \& Onslow, M. (2006). Altered auditory feedback and the treatment of stuttering: A review. Journal of Fluency Disorders, 31, 71-89.

Ling, D. (2002). Speech and the hearing-impaired child: theory and practice. Washington DC: Alexander Graham Bell Association for the Deaf and Hard of Hearing. 
MacKay, D. G. (1968). Metamorphosis of a critical interval: Age-linked changes in the delay in auditory feedback that produces maximal disruption of speech. Journal of the Acoustical Society of America, 43(4), 811-821.

MacLeod, J., Kalinowski, J., Stuart, A., \& Armson, J. (1995). Effect of single and combined auditory feedback on stuttering frequency at two speech rates. Journal of Communication Disorders, 28, 217-228.

Maraist, J. A., \& Hutton, C. (1957). Effects of auditory masking upon the speech of stutterers. Journal of Speech and Hearing Disorders, 22(3), 385-389.

Martin, R. R., Haroldson, S. K., \& Triden, K. (1984). Stuttering and speech naturalness. Journal of Speech and Hearing Disorders, 49, 53-58.

Max, L., Guenther, F. H., Gracco, V. L., Ghosh, S. S., \& Wallace, M. E. (2004). Unstable or insufficiently activated internal models and feedbackbiased motor control as source of dysfluency: A theoretical model of stuttering. Contemporary Issues in Communication Science and Disorders, $31,105-122$.

Natke, U. (2000). Reduction of stuttering frequency using frequency-shifted and delayed auditory feedback. Folia Phoniatrica, 52, 151-159.

Naylor, R. (1953). A comparative study of methods of estimating severity of stuttering. Journal of Speech and Hearing Disorders, $18,30-37$.

O’Donnel, J. J., Armson, J., \& Kiefte, M. (2008). The effectiveness of SpeechEasy during situations of daily living. Journal of Fluency Disorders, $33,99-119$.

Onslow, M. (2001). Frequency altered feedback as an alternative to 'prolonged speech' techniques for the control of stuttered speech. International Journal of Language and Communication Disorders, 36, 409-411.

Packman, A., \& Onslow, M. (1998). The behavioral data language of stuttering. In A. K. Corders \& Ingham (Eds.), Treatment efficacy for stuttering: A search for empirical bases (pp. 213-242). San Diego, California: Singular Publishing Group.

Perkins, W. H., Bell, J., Johnson, K. L., \& Stochs, J. (1979). Phone rate and the effective planning time hypothesis of stuttering. Journal of Speech and Hearing Research, 22, 747-755.

Postma, A. (2000). Detection of errors during speech production: A review of speech monitoring models. Cognition, 77, 97-131.

Postma, A., \& Kolk, H. H. J. (1992). The effects of noise masking and required accuracy on speech errors, dysfluencies, and self-repairs. Journal of Speech and Hearing Research, 35, 537-544.

Riley, G. D. (1994) Stuttering severity instrument for children and adults-third edition (SSI-3). Austin TX: Pro-Ed.

Roach, P. (2000). English phonetics and phonology: A practical course (3rd ed.). Cambridge: Cambridge University Press.

Salemlin, R., Schnitzler, A., Schmitz, F., Jancke, L., Witte, O. W., \& Freund, H. J. (1998). Functional organization of the auditory cortex is different in stutterers and fluent speakers. Neuroreport, 9, 2225-2229.

Soderberg, G. A. (1969). Delayed auditory feedback and the speech of stutterers: A review of studies. Journal of Speech and Hearing Disorders, 34(1), 20-29.

Stuart, A., \& Kalinowski, J. (2004). The perception of speech naturalness of post-therapeutic and altered auditory feedback speech of adults with mild and severe stuttering. Folia Phoniatrica et Logopaedica, 56(6), 347-357.

Stuart, A., Kalinowski, J., Armson, J., Stenstrom, R., \& Jones, K. (1996). Fluency effect of frequency alterations of plus/minus onehalf and one-quarter octave shifts in auditory feedback of people who stutter. Journal of Speech and Hearing Research, 39, 396401.

Stuart, A., Kalinowski, J., \& Rastatter, M. P. (1997). Effect of monaural and binaural altered auditory feedback. Journal of the Acoustical Society of America, 101(6), 3806-3809.

Stuart, A., Kalinowski, J., Rastatter, M. P., \& Lynch, K. (2002). Effect of delayed auditory feedback on normal speakers at two speech rate. Journal of the Acoustical Society of America, 111(5), 2237-2241.

Stuart, A., Kalinowski, J., Rastatter, M. P., Saltuklaroglu, T., \& Dayalu, V. N. (2004). Investigation of the impact of altered auditory feedback in-theear devices on the speech of people who stutter: Initial fitting and 4-month follow-up. International Journal of Language and Communication Disorders, 39(1), 93-113.

Teesson, K., Packman, A., \& Onslow, M. (2003). The lidcombe behavioral data language of stuttering. Journal of Speech, Language, and Hearing Research, 46(1), 1009-1015.

Van Borsel, J., Reunes, G., \& Van den Bergh, N. (2003). Delayed auditory feedback in the treatment of stuttering: Clients as consumers. International Journal of Language and Communication Disorders, 38(2), 119-129.

Van Riper, C. (1971). The nature of stuttering. Englewood Cliffs: Prentice-Hall, Inc.

Venkatagiri, H. S. (2005). Recent advances in the treatment of stuttering: A theoretical perspective. Journal of Communication Disorders, 38(5), 375-393.

Watkins, K. E., Smith, S. M., Davis, S., \& Howell, P. (2008). Structural and functional abnormalities of the motor system in developmental stuttering. Brain, 131, 50-59.

Wingate, M. E. (1970). Stuttering and audition. Journal of Speech and Hearing Research, 13, 861-873.

Yairi, E. (2007). Subtyping stuttering I: A review. Journal of Fluency Disorders, 32, 165-196.

Young, M. A. (1980). Comparison of stuttering frequencies during reading and speaking. Journal of Speech and Hearing Research, 23, $210-222$.

Zimmerman, S., Kalinowski, J., Stuart, A., \& Rastatter, M. P. (1997). Effect of altered auditory feedback on people who stutter during scripted telephone conversations. Journal of Speech Language and Hearing Research, 40, 1130-1134.

Elena Antipova is currently working as a Speech Language Therapist in Oaklynn School for children with special needs (Auckland, New Zealand). She has a MSc (Speech Science) from the University of Auckland, New Zealand and Diploma of Speech and Language Therapy awarded in 1993 by Samara State Pedagogical University, Samara, Russia.

Associate Professor Suzanne Purdy heads Speech Science in the Department of Psychology at the University of Auckland. Dr. Purdy has a MSc (Psychology) from the University of Auckland, a Diploma of Audiology from the University of Melbourne and a PhD (speech pathology and audiology) from the University of Iowa. 
Marjorie Blakeley is currently an Honorary Research Fellow in the Department of Psychology, University of Auckland, associated with the Discipline of Speech Science. Since 2003, she has been involved in private practice as a consultant in speech-language therapy. From 1992/2000 she was Clinical Manager, BSLT Programme, Department of Speech-Language Therapy, University of Canterbury.

Shelley Williams is a Senior Speech and Language Therapist with Auckland District Health Board and Honorary Lecturer at the University of Auckland. She has many years experience in the stuttering field and has been a co-investigator examining efficacy of the Lidcombe program for preschool children. 